\title{
ADERÊNCIA DE CURSOS DE GRADUAÇÃO EM ENFERMAGEM ÀS DIRETRIZES CURRICULARES NACIONAIS NA PERSPECTIVA DO SISTEMA ÚNICO DE SAÚDE
}

\author{
Tack in the nursing graduate courses according to the curriculum national guidelines in \\ the perspective of the sistema único de saúde
}
Adherencia de cursos de graduación en enfermería a las directrices curriculares nacionales en la perspectiva del sistema único de salud

Josicelia Dumêt Fernandes ${ }^{1}$

Raissa Millena Silva Florencio ${ }^{4}$
Rosana Maria de Oliveira Silva²

Lázaro Souza da Silva ${ }^{5}$
Giselle Alves Teixeira ${ }^{3}$

Lyra Cândida Calhau Rebouças ${ }^{6}$

\section{RESUMO}

0 estudo objetivou apreender, nas percepções de discentes de cursos de graduação em enfermagem, o registro da coerência didáticopedagógica desses cursos com as Diretrizes Curriculares Nacionais do Curso de Graduação em Enfermagem, na perspectiva do Sistema Único de Saúde (SUS). Estudo qualitativo, de caráter exploratório descritivo, que teve como cenário quatro cursos de graduação em enfermagem de uma cidade do nordeste do Brasil. Os sujeitos foram discentes que, por meio de entrevistas, indicaram que a articulação entre as instituições formadoras e o SUS se dá no momento da realização das práticas, assim como no ensino dos princípios e diretrizes do SUS, nos componentes curriculares e, ainda, nas atividades desenvolvidas nos projetos de pesquisa e extensão; outros indicaram, também, que não percebem tal articulação no seu processo formativo. 0 resultado do estudo revelou a necessidade de fortalecimento do processo de formação do enfermeiro nos cenários de práticas na rede de serviços de saúde.

Palavras-chave: Educação em Enfermagem. Currículo. Sistema Único de Saúde.

\begin{abstract}
The study aimed to grasp the record of the didactic-pedagogic coherence in nursing graduate courses with the National Curriculum Guidelines for Nursing in the Unified Public Health System (SUS - Sistema Unico de Saude) perspective, according to students perceptions. The exploratory/descriptive qualitative study took place in four nursing graduate programs in a northeastern city in Brazil. The subjects were students who, through interviews, have indicated that the relationship among the institutions that form the SUS takes place at the time of practices implementations, as well as in the teaching of the principles and guidelines from SUS, moreover, in the curricular activities and in the research and extension projects. Others have also mentioned not realizing such relationship in their academic process. The study results revealed the need of strengthen the nursing education process towards the proper health services system.
\end{abstract}

Keywords: Education, Nursing. Curriculum. Unified Health System

\section{Resumen}

El estudio tiene como objetivo la comprensión, la percepción de los alumnos de los cursos de licenciatura en enfermería, el registro de la coherencia didáctica y pedagógica de estos cursos con las Directrices Curriculares Nacionales para la Graduación en Enfermería, en la perspectiva del Sistema Único de Salud (SUS). Estudio cualitativo y exploratorio, descriptivo, teniendo como escenario cuatro cursos de graduación en enfermería en una ciudad del noroeste de Brasil. Los sujetos eran estudiantes que, a través de entrevistas, indicaron que el vínculo entre las instituciones de formación y el SUS se lleva a cabo en el momento de la aplicación de prácticas, así como la enseñanza de los principios y directrices del SUS en los componentes del plan de estudios y también las actividades en los proyectos de investigación y extensión, otros han señalado, también, que no se dan cuenta de esta vinculación en su proceso formativo. Los resultados del estudio evidencian la necesidad de fortalecer el proceso de formación de enfermería en los escenarios dentro de la red de servicios de salud.

Palabras clave: Educación en Enfermería. Curriculum. Sistema Único de Salud.

\footnotetext{
'Doutora. Professora Titular da Escola de Enfermagem da Universidade Federal da Bahia. Líder do Grupo de Estudos e Pesquisas em Educação, Ética e Exercício de Enfermagem da Escola de Enfermagem da Universidade Federal da Bahia. Salvador-BA. Brasil. E-mail: dumet@urba.br; ${ }^{2}$ Mestre. Professora Assistente da Escola de Enfermagem da Universidade Federal da Bahia. Aluna do Curso de Doutorado do Programa de Pós-Graduação da Escola de Enfermagem da Universidade Federal da Bahia. Membro do Grupo de Estudos e Pesquisas em Educação, Ética e Exerćíio de Enfermagem da Escola de Enfermagem da Universidade Federal da Bahia. Salvador - BA. Brasil. E-mail: rosanaosilva@hotmail.com; ${ }^{3}$ Mestre. Aluna do Curso de Doutorado do Programa de Pós-Graduação da Escola de Enfermagem da Universidade Federal da Bahia. Membro do Grupo de Estudos e Pesquisas em Educação, Ética e Exercício de Enfermagem da Escola de Enfermagem da Universidade Federal da Bahia. Salvador - BA. Brasil. E-mail- giselletnf@hotmail.com; ${ }^{4}$ Especialista. Aluna do Curso de Mestrado do Programa de Pós-Graduação da Escola de Enfermagem da Universidade Federal da Bahia. Membro do Grupo de Estudos e Pesquisas em Educação, Ética e Exercício de Enfermagem da Escola de Enfermagem da Universidade Federal da Bahia. Salvador - BA. Brasil. E-mail- raissaflorencio@yahoo.com.br; ${ }^{5}$ Graduando em Enfermagem. Bolsista do Programa de Iniciação Cientíica da Universidade Federal da Bahia. Membro do Grupo de Estudos e Pesquisas em Educação, Ética e Exercício de Enfermagem da Escola de Enfermagem da Universidade Federal da Bahia. Salvador - BA. Brasil. E-mail- lazo_Iss@hotmail.com; ${ }^{\circ}$ Mestre. Professora Assistente da Universidade Estadual do Sudoeste da Bahia. Aluna do Curso de Doutorado do Programa de Pós-Graduação da Escola de Enfermagem da Universidade Federal da Bahia. Membro do Grupo de Estudos e Pesquisas em Educação, Ética e Exercício de Enfermagem da Escola de Enfermagem da Universidade Federal da Bahia. Salvador - BA. Brasil. E-maillyracalhau@gmail.com
} 


\section{INTRODUÇÃO}

O Sistema Único de Saúde (SUS) oferece suporte para a efetivação da política de saúde no Brasil, traduzindo em ação os princípios e diretrizes desta política. Foi instituído na Constituição de 1988 e normatizado pela Lei n. ${ }^{8} 8.080 / 90$ Lei Orgânica da Saúde ${ }^{1}$, tendo como princípios doutrinários a universalidade, integralidade e equidade e, como diretrizes organizativas, a descentralização em comando único, a hierarquização e regionalização dos serviços, além do controle social.

Esses princípios e diretrizes buscam viabilizar a substituição do modelo hegemônico biologicista, individual, centrado no médico e na doença por um modelo de atenção à saúde de natureza includente, universal, equânime e integral; um modelo de atenção à saúde centrado na vigilância e prevenção de agravos, com ações de promoção, prevenção, recuperação e reabilitação da saúde e com ativa participação e controle social.

Esse modelo implica o desafio de redirecionar as práticas de prestação de serviços e de formação de profissionais de saúde para 0 atendimento às necessidades de saúde individuais e coletivas da população.

Um dos objetivos/atribuições do SUS, assegurado por Lei' é a participação na formulação e na execução da política de formação e desenvolvimento de recursos humanos para a saúde, que, por sua vez, demanda uma mudança do perfil dos recursos humanos na área da saúde, o que requer uma forte política intersetorial entre os setores saúde e educação.

Nesse contexto, concretizou-se, em 7/8/2001, o Parecer 1133 do CNE/CES ${ }^{2}$, que veio reforçar a necessidade da articulação entre Educação Superior e Saúde, objetivando a formação geral e específica dos egressos/profissionais com ênfase na promoção, prevenção, recuperação e reabilitação da saúde.

Após esse Parecer, foi aprovada a Resolução CNE/CES № 03 de 7/11/2001 ${ }^{3}$, que definiu as Diretrizes Curriculares Nacionais para o Curso de Graduação em Enfermagem (DCN/ ENF). Essa Resolução, evidenciando a necessidade de articulação entre os setores educação e saúde, traz indicadores de mudanças processuais na formação do enfermeiro que se apresentam como grandes desafios às Instituições de Educação Superior.

Essas mudanças reafirmam a necessidade e o dever dessas instituições de formarem profissionais enfermeiros capacitados para atender às necessidades ampliadas de saúde da população com ênfase no Sistema Único de Saúde (SUS) e assegurar a integralidade da atenção e a qualidade e humanização do atendimento ${ }^{3}$. Elas abrem possibilidades para a transformação do perfil dos futuros profissionais, por meio da adoção de estratégias construídas com base nos princípios e diretrizes desse Sistema, além de estarem fundamentadas no conceito ampliado de saúde; na utilização de metodologias ativas de ensino-aprendizagem, que considerem o trabalho em saúde como eixo estruturante das atividades; no trabalho multiprofissional e transdisciplinar da equipe de saúde; na integração entre o ensino e os serviços de saúde; e no aperfeiçoamento da atenção integral à saúde da população ${ }^{4}$.

Sob essa ótica, as DCN/ENF possibilitam uma sólida formação de acordo com o estágio de desenvolvimento do conhecimento na área, permitindo ao graduado enfrentar as rápidas mudanças do conhecimento e seus reflexos no mundo do trabalho. Possibilitam, ainda, a construção de um perfil acadêmico e profissional que levem, por meio de perspectivas e abordagens contemporâneas, ao desenvolvimento de competências e habilidades que possam fundamentar a formação do profissional crítico-reflexivo, transformador da realidade social e agente de mudanças, destacando a importância da diversidade de cenários de aprendizagem, com ênfase no SUS e na realidade locorregional para proporcionar a integralidade das ações de qualidade e humanas de enfermagem ${ }^{4}$. Tornam possível, pois, a formação de profissionais inseridos na dinâmica do sistema, contextualizados com a realidade da sociedade e sensibilizados para o trabalho em equipes multiprofissionais ${ }^{5}$.

Em situações de trabalho no fazer cotidiano do processo de formação do enfermeiro, contudo, temos observado a existência de desafios na construção de uma articulação entre as instituições formadoras e a rede SUS, onde fica evidente um descompasso entre a formação profissional e as reais necessidades desse Sistema e, consequentemente, com as DCN/ ENF.

Perante essa observação, levantamos a seguinte questão: Como se dá a aderência dos cursos de graduação em enfermagem às DCN/ENF, na perspectiva do SUS?

Buscando respostas a esse questionamento, este trabalho tem como objetivo apreender, nas percepções de discentes de cursos de graduação em enfermagem, o registro da coerência didático-pedagógica desses cursos com as DCN/ ENF, na perspectiva do SUS.

0 presente estudo se justifica, pois, pela necessidade de realização de estudos que possam contribuir na formação do perfil de futuros profissionais, assim como no planejamento e implementação de políticas de formação e inserção profissional do enfermeiro, construídas à luz dos princípios e diretrizes do sistema público de saúde.

\section{METODOLOGIA}

Trata-se de uma pesquisa descritiva e analítica com abordagem qualitativa, que buscou apreender, nas percepções de discentes de cursos de graduação em enfermagem, o registro da coerência didático-pedagógica desses cursos com as DCN/ ENF, na perspectiva do SUS.

A opção para o alcance do objetivo pela percepção de discentes foi pautada no entendimento de que estes têm uma importante relevância em relação ao seu processo de 
ensino-aprendizagem, pois deixam de ser meros coadjuvantes e passam a configurar-se como transformadores da sua realidade e detentores de conhecimentos que devem ser valorizados ${ }^{3}$.

Entendemos que o estudante crítico-reflexivo terá mais chances, ao longo do curso, de identificar os meandros de sua formação, como também propor discussões sobre a capacidade que o curso tem de prepará-lo para o mundo do trabalho no âmbito do SUS.

Com esse entendimento, acreditamos que as percepções dos futuros profissionais privilegiam uma análise apurada a respeito da coerência didático-pedagógica dos cursos de enfermagem com as DCN/ENF, na perspectiva do SUS.

Participaram do estudo, 13 discentes de quatro Instituições de Ensino Superior (IES) que ofereciam cursos de graduação em enfermagem, públicas e privadas, de uma cidade da região nordeste do Brasil. Como critério de inclusão, os discentes deveriam estar cursando o componente curricular Estágio Curricular Supervisionado I ou ll e aceitar participar do estudo assinando o Termo de Consentimento Livre e Esclarecido.

Em um universo de $19 \mathrm{IES}$, à época da coleta dos dados, oito tinham alunos que atendiam o critério acima mencionado, quatro das quais aceitaram participar do estudo.

Após autorização do Coordenador da IES e parecer de aprovação de nº 016.2010 do Comitê de Ética em Pesquisa da Escola de Enfermagem da Universidade Federal da Bahia, os pesquisadores mantiveram contato com os discentes que agendaram os encontros conforme suas disponibilidades. A coleta de dados ocorreu na própria instituição ou nos campos de pratica/estagio onde os entrevistados estavam em atividade, seguindo o critério de exaustão/saturação dos dados.

0 instrumento para coleta de dados foi a entrevista semiestruturada composta pelas questões norteadoras: (a) fale sobre o SUS; (b) como você percebe a inserção das suas atividades discentes nos serviços oferecidos pelo SUS; (c) como você percebe a articulação do seu curso com os princípios do SUS.

Os depoimentos foram gravados com a utilização de gravadores de voz digitais, após permissão dos sujeitos, estando os mesmos cientes do sigilo, confidencialidade, garantia de privacidade e de anonimato, conforme Resolução 196/96 do Conselho Nacional de Saúde. Para a análise dos dados foi utilizada a Análise de Conteúdo de Bardin 6 . Após a realização das entrevistas e sua transcrição, foram realizadas leituras das falas dos entrevistados. Em seguida, as falas foram divididas quanto às questões do instrumento correspondente, recortadas e agrupadas em unidades de contexto, que contemplavam o objetivo da pesquisa. Posteriormente as falas foram coligidas quanto ao número de repetições e sentido semelhantes, analisadas, interpretadas e discutidas à luz da literatura existente sobre o tema.

\section{RESULTADOS}

Após a análise das unidades de contexto, as falas foram agrupadas em duas categorias, a saber: "Articulação do processo de formação do enfermeiro com a rede SUS" e "(In)coerência didático-pedagógica dos cursos de enfermagem com as DCN/ENF, na perspectiva do SUS".

\section{Articulação do processo de formação do enfermeiro com a rede SUS}

A análise das falas dos sujeitos revelou que a articulação entre o processo de formação e a rede do SUS dá-se, entre outros, no momento em que as práticas de ensino se realizam nos cenários de aprendizagem em saúde, conforme ilustrado a seguir:

[...] essa articulação, eu diria que é uma articulação forte, no sentido de que nós atuamos diretamente nos cenários das práticas em saúde, com uma carga horária densa que toma maior parte do curso.... (E.10)

Eu acho bem articulada porque nós podemos [...] atuar em todo o processo como a atenção primária, secundária e terciária [...]. (E.4)

Essas falas encontram respaldo no inciso VI do Art. $5^{\circ}$ da Resolução 03/2001 que trata das DCN/ENF ao explicitar como Habilidades e Competências específicas para o enfermeiro, dentre outras, o reconhecimento da saúde como direito e condições dignas de vida, além da atuação de forma a garantir a integralidade da assistência, entendida como conjunto articulado e contínuo das ações e serviços preventivos e curativos, individuais e coletivos, exigidos para cada caso em todos os níveis de complexidade do sistema ${ }^{3}$.

Outro aspecto levantado pelos sujeitos entrevistados foi a diversidade de cenários de aprendizagem como estratégia para superar a formação voltada para o conhecimento fisiopatológico e distante da realidade sócio-sanitária e da prestação do cuidado na rede SUS.

[...] todas as práticas que nós desenvolvemos durante o curso foram nos espaços de atuação do SUS... e foram muito os espaços de aprendizagem que usamos. [...] Existe uma articulação, sim. (E.11)

Bom, tem uma total interligação, [...] nós fazemos os estágios em hospitais-escola, na rede básica, em postos conveniados, então é totalmente SUS. [...]. (E.6)

Essas falas encontram suporte no inciso XVI do Art. $5^{\circ}$ das DCN/ENF quando explicita que a formação do enfermeiro 
deve dotar o profissional de conhecimentos requeridos para o exercício de competências e habilidades específicas, em que se destaca, dentre outras, a necessidade de atuação nos diferentes cenários da prática profissional, considerando os pressupostos dos modelos clínico e epidemiológico ${ }^{3}$.

Entende-se, destarte, que a diversidade de cenários de aprendizagem se constitui em uma estratégia de aderência às DCN/ENF.

A formação pretendida para o enfermeiro implica, pois, na utilização dos espaços de aprendizagem nas unidades básicas de saúde, unidades de saúde da família e hospitais secundários e terciários como essenciais para atender as DCN/ENF.'

Outro aspecto colocado pelos sujeitos da pesquisa, e que estão em consonância com as DCN/ENF, foi a importância de engajamento do estudante no processo de trabalho do enfermeiro, nos cenários das práticas em saúde, conforme fragmentos de falas, a seguir:

\section{[...] passamos por vários serviços da rede SUS, O} que favoreceu a nossa integração nesses serviços [...] pudemos participar do processo de trabalho do enfermeiro, do processo de tomada de decisões e do trabalho em equipe..... (E.9)

Eu posso dizer que não tive um ensino livresco [...] tive um ensino articulado com a prática dos serviços de saúde. (E.3)

Outro aspecto evidenciado pelos sujeitos foi em relação aos projetos de pesquisa e às atividades de extensão, que assumem um papel importante no desenvolvimento crítico e reflexivo do aluno, sendo outra forma de perceber a articulação da instituição formadora com os princípios e diretrizes do SUS, como nos depoimentos que se seguem:

Sim, [...] os grupos de pesquisa desenvolvem pesquisas nos cenários do SUS. Eu participo do PET, Programa de Educação Tutorial, a gente faz pesquisa junto a um PSF. (E.11)

[...] tem o grupo de Idoso que faz atividades físicas. [...] Fazemos atividades de prestação de serviços, através de campanhas de vacina promovidas pelo SUS. [...]. (E.3)

[... na parte da saúde pública os trabalhos de extensão são muitos... todos através do SUS. (E.5)

[...] tem várias atividades de pesquisa lá que são realizadas. Tem um projeto para as parteiras da Chapada Diamantina. [...]. (E.6)
Essas falas apontam para a percepção de que as IES estudadas apresentam alguma aproximação com as DCN/ENF, que no seu Art. $8^{\circ}$ diz que as IES deverão criar mecanismos de aproveitamento de conhecimentos, adquiridos pelo estudante, por meio de estudos e práticas independentes, presenciais e/ ou a distância, a saber: monitorias e estágios; programas de iniciação científica; programas de extensão; estudos complementares e cursos realizados em outras áreas afins ${ }^{3}$.

A articulação do processo de formação do enfermeiro com as DCN/ENF, na perspectiva do SUS, foi percebida, também, a partir dos componentes curriculares que discutem os princípios, diretrizes e abrangência do SUS, especificamente os conteúdos da saúde pública, nos quais é discutida a formação de atitudes evalores preconizadas pelo SUS, conforme ilustram as falas:

[...] a partir do $3^{\circ}$ semestre, praticamente todos os conteúdos da área profissionalizante tiveram assuntos com aderência ao SUS, porque nossa formação é muito voltada, mesmo, para o SUS. (E.7)

[...] Nós tivemos conteúdos de Saúde Coletiva le Saúde Coletiva Il, [...] Políticas Públicas, [...] todas as nossas matérias especificas sempre inerentes ao SUS. [...] A nossa formação é mesmo voltada para o SUS. (E.5)

[...] eu diria que a maioria dos conteúdos curriculares têm a ver com o SUS. (E.10)

Essas falas apontam para a percepção de que os depoentes reconhecem a coerência do processo de formação do enfermeiro com as DCN/ENF, na perspectiva do SUS acontecendo a partir dos conteúdos curriculares.

Ficou evidente, também, que competências e habilidades relacionadas à formação de atitudes e valores preconizados pelo SUS e requeridas para o exercício profissional estão sendo desenvolvidas em conformidade com as DNC/ENF, de acordo com as ilustrações a seguir.

É ter uma formação generalista com responsabilidade, humanidade, conhecimento técnico cientifica e ética no trabalho. [...]. (E.6)

É preciso ter empatia,[...] você tem que ser humilde, e além de tudo você tem que ter ética,[...] acho que o principal de tudo é ética. [...]. (E.13)

[...] Eu acho que a responsabilidade, compromisso, conhecimento científico e sensibilidade. (E.8)

[...] ser flexível, respeitar o saber do outro, respeitar o outro, a cultura, o saber popular, 
oferecer ao sujeito, ao outro a possibilidade de tomar decisões [... ] é, acima de tudo, compromisso com a saúde. (E.11)

Essas falas encontram suporte nas DCN/ENF, que, no inciso I do seu Art. $3^{\circ}$, explicita que o futuro egresso do Curso de Graduação em Enfermagem deve ser um "profissional com formação generalista, humanista, crítica e reflexiva"; o curso deve preparar o futuro egresso para o exercício de Enfermagem, "com base no rigor científico e intelectual e pautado em princípios éticos"; deve ser "capaz de conhecer e intervir sobre os problemas/situações de saúde-doença mais prevalentes no perfil epidemiológico nacional, com ênfase na sua região de atuação, identificando as dimensões biopsicossociais dos seus determinantes"; deve ainda estar "capacitado a atuar, com senso de responsabilidade social e compromisso com a cidadania, como promotor da saúde integral do ser humano"3.

(In)Coerência didático-pedagógica dos cursos de enfermagem com as DCN/ENF, na perspectiva do SUS

Nesta categoria foram agrupados os depoimentos de sujeitos que não perceberam uma articulação relevante entre 0 processo de formação e o SUS. Em alguns casos, os discentes simplesmente negam a existência dessa articulação e buscam explicar essa realidade a partir da percepção gerada nos cenários didático-pedagógicos.

Ao buscar formar profissionais com visão crítica e reflexiva, as instituições fornecem aos alunos valiosas ferramentas para discernirem sobre a realidade mais ampla, de modo que possam perceber as incongruências de sua formação. Assim, foi possível registrar, a partir dos depoimentos, a percepção dos discentes de que os cursos apresentam, sob diversos aspectos, contradições importantes entre as práticas pedagógicas e a formação do egresso. Se por um lado o curso disponibiliza o conhecimento técnico-científico necessário para 0 aluno atuar no SUS, por outro, não valoriza efetivamente as peculiaridades da formação para o sistema de saúde vigente.

A articulação mesmo é só em colocar nós alunos nos campos de prática, sem uma preocupação com a nossa formação. [...] (E.5)

Eu acho que relação atualmente ela é muito mais utilitarista da faculdade com o sistema único de saúde, também com a faculdade em aproveitar uma mão de obra barata que é.... somos nós estudantes [...] (E.7)

[...]Agente tem que colaborar [...]com o nosso trabalho, mão de obra através do estágio. Não vejo o retorno do ponto de vista pedagógico (E.3)
Essa percepção de desarticulação entre a IES e o SUS acarreta no discente uma insegurança quanto ao seu fazer, um sentimento de impotência quanto ao ser enfermeiro. $E$ essas inconsistências percebidas pelo aluno possivelmente interferirão no seu agir nos cenários das práticas em saúde, conforme ilustrado nas falas a seguir.

Nem como enfermeira, nem no SUS. [...] eu ainda não me acho preparada [....]. (E.8)

Eu acho que eu preciso treinar bastante ainda... eu estou um pouco insegura, até porque minhas práticas foram bem poucas [...] eu tive mais sala de aula... eu preciso de um pouco mais de prática [...]. (E.13)

Além desse sentimento de despreparo para atuar nos espaços das práticas, manifestado pelos discentes, ficou aparente a percepção de um distanciamento entre a teoria e as prática pedagógicas, como é possível detectar nas falas abaixo:

[...] na prática não é aquilo que a gente vê na teoria, a teoria é bem diferente da prática. (E5)

[...] A realidade é muito diferente da teoria.(E8)

Outro aspecto constatado em algumas falas foi uma dificuldade em entrar e estar nos campos de prática, ou seja não existe articulação entre o ensino e o serviço:

Não adianta a Escola de Enfermagem querer se articular com o serviço, querer trazer um pouco o que ela tem de teoria pra estar atualizando se as vezes o campo de prática está fechado, está com suas portas fechadas pra Universidade... deve ser uma via de mão dupla [...], o serviço precisa querer a Universidade dentro dele e a Universidade precisa querer também entrar nesse serviço [...]. (E.8)

Sinceramente eu acho que a articulação entre a faculdade e a rede SUS deixa muito a desejar ... se realmente tivesse articulação não encontraríamos dificuldades em entrar nos campos de prática. ...Falta um pouquinho mais de articulação entre a universidade e os serviços. (E.5)

Apesar de todas as possibilidades existentes nos espaços de integração ensino-serviço, é inquietante perceber que a formação em saúde - que utiliza os serviços da rede pública de saúde como campo privilegiado para as atividades práticas que compõem essa formaçã̃o, seja na modalidade de 
estágio curricular ou na de aula prática — tem uma inserção nesses serviços que se caracteriza por um relativo distanciamento, um tratamento de certa forma cerimonioso entre os envolvidos, no qual as críticas que tenham ao outro não encontram canais adequados de expressão. Dessa forma, sem o diálogo esperado, limitam-se as possibilidades de um fazer diferenciado, que assuma concepções acerca do cuidado, dos processos e organização do trabalho, da gestão e da escuta do usuário ${ }^{7}$.

Sendo assim, é preciso investir na sensibilização dos atores inseridos nos cenários onde se desenvolvem os cuidados e o processo de ensino-aprendizagem. Operacionalmente, a universidade deve se preocupar em identificar necessidades dos serviços e cenários de prática, estabelecendo pactos de contribuição docente/discente para tais serviços. Além disso, em contrapartida, é fundamental a participação de profissionais dos serviços e usuários nas discussões educacionais de formação na área da saúde?

Outro aspecto destacado pelos discentes são os vários contratempos encontrados pelas instituições para garantir os espaços de práticas, o que dificulta o desenvolvimento dessa atividade, conforme ilustrado, a seguir:

[...] a gente tem dificuldade em ir num estagio, [...] começa o semestre e temos dificuldade pra conseguir os estagio... a gente tem dificuldade na própria faculdade em relação à administração, por exemplo, da instituição do local onde a gente está estagiando que é do SUS. (E.13)

[...] ]até certo tempo foi boa, mas, agora, ultimamente, está tendo muita dificuldade pra conseguir o campo de prática. [...] (E.12)

Ficou evidente na fala dos discentes a dificuldade em conseguir campos de práticas devido ao descompasso entre o número de cursos de enfermagem e os cenários das práticas em saúde. Como o número de faculdades é grande e os cenários das práticas são poucos, observa-se uma "superlotação" desses espaços, o que interfere no desenvolvimento do processo de formação dos alunos. Considerando essa dificuldade, alguns cursos desenvolvem algumas atividades em laboratórios virtuais, o que leva a um distanciamento do aluno com o cliente/comunidade, tornando o processo de formação livresco, sem uma aderência às práticas em saúde.

Há que se considerar que existe entre a teoria e a prática uma relação permanente e dinâmica, sustentando-se mutuamente, e é por meio dessa relação dialógica que elimina-se a dicotomia opositiva entre elas. Contudo, esse diálogo há que se expandir até o espaço real onde as práticas de saúde acontecem, já que interessa para a formação profissional não só o aprendizado da técnica, mas o desenvolvimento de um conjunto de competências e habilidades que só se darão no convivio com o outro, na participação nas atividades de rotina, na vivência da realidade onde futuramente atuará.

\section{DISCUSSÃO}

A articulação entre as instituições formadoras e a rede SUS se constitui numa realidade do incremento das atividades discentes na rede de serviços. Para tanto, é preciso desvelar o conhecimento que os atores envolvidos têm dessa articulação. Nesse sentido, percebe-se que a transformação no contexto da Educação em Enfermagem se dá no instante em que professor e aluno percebem a importância de seus papéis no processo de ensinar/aprender, tornando-se mais conscientes, responsáveis em aprender, valorizando a comunicação, que possibilita a articulação das ações e a integração dos envolvidos, movidos pelo agir comunicativo, o que gera condições de um membro interagir com o todo 8 .

As informações levantadas, a partir das entrevistas com os sujeitos da presente investigação, mostraram que a formação do enfermeiro vem sofrendo alterações em seu modelo pedagógico, buscando reorientá-lo para as necessidades da sociedade e do SUS. Mostraram, também, a presença de contradições na percepção dos discentes quanto à coerência didático-pedagógica dos cursos de graduação em enfermagem no que diz respeito à sua aderência às $D C N / E N F$, na perspectiva do SUS.

Os sujeitos perceberam a existência da articulação entre seu curso e as DCN/ENF, na perspectiva do SUS, ao contrário de outros que não viram tal articulação.

Observamos que a articulação do processo de formação com a rede SUS é percebida quando da realização das práticas nos serviços da rede. Mas a formação discente vai além disso. Ela engloba aspectos de produção de subjetividade, produção de habilidades técnicas e de pensamento e 0 adequado conhecimento do SUS9.

Os estudantes perceberam, ainda, que a articulação do curso com os princípios do SUS ocorre, também, pelos projetos de pesquisa e extensão realizados nos serviços da rede, favorecendo a articulação entre o ensino, pesquisa e extensão/ assistência, além de garantir um ensino crítico, reflexivo e criativo que leva em conta a evolução epistemológica dos modelos explicativos do processo saúde-doença ${ }^{3}$.

Esses projetos instrumentalizam o aluno na resolução dos problemas, permitem o desenvolvimento da criatividade e a continuidade dos estudos, além de formação de habilidades de construir seu raciocínio, incentivar a ter sua própria opinião e reflexão e a transformar a necessidade em motivação, embasando-o na tomada de decisões administrativas e clínicas e incentivando-o a prosseguir com sua formação acadêmica ${ }^{10}$.

As discussões dentro dos componentes curriculares sobre o SUS serviram para dar aos estudantes a dimensão da 
interdependência entre eles e os cursos. Várias disciplinas destacaram-se no papel de articuladoras ao abrirem espaço para debater e refletir sobre o sistema de saúde vigente. No Art. 5, Parágrafo Único, das DCN/ENF, a formação do Enfermeiro deve atender às necessidades de saúde, com ênfase no Sistema Único de Saúde (SUS) e assegurar a integralidade da atenção e a qualidade e humanização da assistência ${ }^{3}$. $\mathrm{Na}$ fala dos sujeitos percebemos que os cursos estão de acordo com esse Artigo ao abordarem em seus componentes curriculares os temas pertinentes à saúde na perspectiva do SUS.

Os depoimentos dos sujeitos indicaram que os estudantes entendem a articulação entre seu curso e o SUS quando demonstram um conhecimento autêntico sobre as competências e habilidades que precisam desenvolver para atuar na rede.

Por outro lado, observamos relatos de dificuldades na efetivação da articulação entre as instituiç̃es formadoras e a rede SUS. Alguns depoentes negaram, inclusive, a existência de tal aderência.

A polaridade dessa situação ficou evidente quando os alunos explicitaram o sentimento de que são considerados tão somente mão de obra barata nos campos de práticas. Para esses discentes, há pouca preocupação da universidade com sua formação. Os serviços não são escolhidos como cenários de práticas por refletirem efetivamente os princípios do SUS. Ou seja, os cenários das práticas não são cuidadosamente selecionados, tendo em vista o desenvolvimento, no discente, das competências e habilidades previstas nas DCN/ENF, na perspectiva do SUS.

Os depoimentos relatam que os alunos passam por varias especialidades, realizando diversos procedimentos. Entretanto, não há menção se essas especialidades ou procedimentos convergem para o desenvolvimento de competências e habilidades para atuar no SUS.

Gestores do SUS reclamam que os profissionais vêm com formação inadequada, que os estudantes não são expostos às melhores aprendizagens e que as universidades não têm compromisso com o SUS ${ }^{11}$.

Entretanto, para outros estudiosos, o SUS tem assumido um papel ativo na reorientação das estratégias e, com seu modo de cuidar, tratar e acompanhar a saúde individual e coletiva, tem sido capaz de provocar importantes repercussões nas estratégias e maneiras de ensinar e aprender ${ }^{12}$.

Contudo, é neste mesmo sentido que se apresenta a necessidade de rever os modos de formação para se atuar no sistema de saúde, o que implica o estabelecimento de estratégias para transformar a organização dos serviços e dos processos formativos, das práticas pedagógicas.

Os discentes demonstraram insatisfação com a falta de articulação também quando percebem um distanciamento entre a aprendizagem teórica e a prática, tornando a formação basicamente teórica, na qual os alunos dizem que, apesar do bom aporte teórico, é necessário um bom conteúdo prático para que diminua esse distanciamento a teoria e a prática.

Vale destacar que a prática não é mais compreendida como uma mera atividade, mas como enfrentamento de eventos, pois cada vez mais depende do conhecimento teórico; e que a teoria é tida como um "guia" na orientação dessas práticas, daí a importância da interação e articulação entre elas ${ }^{13}$.

0 estágio curricular é tomado como uma etapa de ampliação do conhecimento reflexivo e do aperfeiçoamento de habilidades em uma situação real. É o momento de junção do saber com o fazer, com vista a conduzir o estudante a um agir profissional mais consciente, crítico e criativo ${ }^{14}$. Entretanto, alguns depoimentos apontam para a falta de articulação do Estágio Curricular com a rede SUS, quando o aluno encontra resistência por parte dos serviços para aceitá-lo nos campos de prática.

Nesse contexto, os alunos acabam sendo prejudicados devido à falta dessa atividade prática, pois, em alguns casos, muitos alunos saem da graduação sem ter tido o contato com a realidade, prejudicando-se com relação à atuação profissional, muito embora o Art. $7^{\circ}$, das DNC/ENF, referir que os cursos de enfermagem são obrigados a incluir no currículo o estágio supervisionado em hospitais gerais e especializados, ambulatórios, rede básica de serviços de saúde e comunidade 3 .

\section{CONSIDERAÇÕES FINAIS}

Vivemos um momento marcado por rápidas e numerosas transformações no mundo da política, da economia, da cultura, da educação e da saúde, que exigem respostas no campo do trabalho e da formação para o trabalho. Nesse sentido, temos que buscar constantemente eixos e estratégias que conduzam o processo de formação, de modo a atender às demandas requeridas pelo atual contexto mundial, nacional e principalmente local.

Atualmente, no campo de formação para a saúde, uma das maiores demandas é a formação de profissionais em consonância com os princípios do Sistema Único de Saúde, profissionais que sejam capazes de enfrentar os desafios apontados nos serviços, com melhor compreensão e consciência crítica da realidade na qual estão inseridos, com transformação das práticas assistenciais, voltadas para a integralidade e justiça social.

0 estudo revelou a necessidade de fortalecimento do processo de formação do enfermeiro nos cenários de práticas na rede de serviços de saúde, onde se dá o desenvolvimento do cuidado e a produção do conhecimento próprio da enfermagem.

É importante que academia e serviço entendam que cada um é fruto de um contexto, com diferentes experiências e diferentes objetivos. No entanto, reconhecê-las, respeitá-las e trabalhá-las tornam-se fatores essenciais para a construção do trabalho em saúde na perspectiva do SUS, uma vez que se faz necessário construir possibilidades de superação das fragmentações no campo do saber e do trabalho. 
Para concluir, torna-se necessário explicitar que 0 presente estudo não se constitui em um trabalho acabado, nem, tampouco, indica caminhos a serem percorridos pelas instituições/escolas/cursos para alcançarem as transformações necessárias. Trata-se apenas da apresentação de parte de uma realidade desafiadora do processo de formação do enfermeiro e sua articulação com as DCN/ENF, na perspectiva do SUS, contribuindo para a melhoria da qualidade desse processo, bem como para o planejamento e implementação de políticas de formação e inserção profissional do enfermeiro, construídas à luz dos princípios e diretrizes do SUS.

\section{REFERÊNCIAS}

1. Lei 8.080 de 19 de setembro de 1990. Dispõe sobre as condições para promoção, proteção e recuperação da saúde, a organização e funcionamento dos serviços correspondentes e dá outras providências. Diário Oficial da República Federativa do Brasil, Brasilia (DF), 20 de setembro de 1990.

2. Ministério da Educação (BR), Conselho Nacional de Educação, Câmara de Educação Superior. Parecer $n^{\circ}$ 1.133, de 07 de agosto de 2001. Dispõe sobre as Diretrizes Curriculares da Medicina, Enfermagem e Nutrição. Brasilia(DF): Ministério da Educação e Cultura; 2001.

3.Ministério da Educação (BR). Conselho Nacional de Educação. Câmara da Educação Superior. Resolução nº 3, de 07 de novembro de 2001. Institui Diretrizes Curriculares Nacionais do Curso de Graduação em Enfermagem. Brasília(DF): Ministério da Educação e Cultura; 2001.

4. Lopes Neto D, et al. Aderência dos Cursos de Graduação em Enfermagem às Diretrizes Curriculares Nacionais. Rev. bras. enferm. 2007 nov; 60(6): 625-34.

5. Fernandes JD, Xavier IM, Ceribelli MIPF, Bianco MHC, Maeda D, Rodrigues MVC. Diretrizes curriculares e estratégias para implantação de uma nova proposta pedagógica. Rev. Esc. Enferm. USP. 2005 dez; 39(4): 443-9.

6. Bardin L. Análise de Conteúdo. Lisboa: Edições 70; 2009.

7. Henriques RLM. Interlocução entre ensino e serviço: possibilidades de ressignificação do trabalho em equipe na perspectiva da construção social da demanda. In: Pinheiro R, Mattos RA, organizadores. Construção social da demanda. Rio de Janeiro(RJ): IMS, UERJ, CEPESC, ABRASCO; 2005.

8. Fernandes CNS. Refletindo sobre o aprendizado do papel de educador no processo de formação do enfermeiro. Rev. Latino-Am. Enfermagem. 2004 jul/aug.; 12 (4): 691-3.

9. Ceccim RB, Feuerwerker LCM. Mudança na graduação das profissões de saúde sob o eixo da integralidade. Cad. saúde pública. 2004 set; 20(5): 1400-10.

10. Palmeira IP, Rodriguéz MB. A investigação científica no curso de enfermagem: uma análise crítica. Esc Anna Nery. 2008 mar; 12(1): 6875.
11. Ceccim RB. Educação Permanente em Saúde:descentralização e disseminação de capacidade pedagógica na saúde. Ciênc. saúde coletiva. 2005 out/dez; 10(4): 975-86.

12. Ferreira RC, Fiorini VML, Crivelaro E. Formação Profissional no SUS: 0 Papel da Atenção Básica em Saúde na Perspectiva Docente. Rev. bras. educ. méd. 2010 abr/jun; 34(2): 207-15.

13. Kuenzer AZ. As relações entre conhecimento tácito e conhecimento científico a partir da base microeletrônica: primeiras aproximações. Educar rev. Curitiba. 2003; 21(2): 43-69.

14. Tavares CMM. Análise crítica de uma experiência de integração do estágio de enfermagem em saúde mental ao Sistema único de saúde. Esc Anna Nery. 2006 dez; 10(4): 740-7. 